\title{
Adaptive Resonant Vibration Control of a Piezoelectric Flexible Plate Implementing Filtered-X LMS Algorithm
}

\author{
Zhi-cheng Qiu and Biao Ma \\ School of Mechanical and Automotive Engineering, South China University of Technology, Guangzhou 510641, \\ PR China
}

(Received 3 July 2013; revised: 15 November 2013; accepted: 2 December 2013)

\begin{abstract}
Vibration in aerospace structures can lead to structural damage. To solve this problem, the implementation of active vibration control must be considered. This paper investigates active vibration control under the persistent resonant excitation of a clamped-clamped piezoelectric plate system. The finite element method (FEM) and ANSYS modal analysis methods are utilized to obtain the dynamics model and mode shapes of the plate. A two-norm criterion is used for optimal placement of piezoelectric sensors and actuators, taking into account the non-controlled modes to reduce spillover problems. A genetic algorithm (GA) is used to search the optimal locations of actuators/sensors. Then, a proportional derivative (PD) control algorithm and a filtered-X least mean square (filtered-X LMS) feedforward control algorithm are designed for the system. Subsequently, numerical simulations with optimal placement of actuators and sensors are carried out to compare the performance of the controllers. Finally, experiments are conducted. The experimental results demonstrate that the designed filtered-X LMS control algorithms can suppress the resonant vibration better than that of the PD control.
\end{abstract}

\section{INTRODUCTION}

Flexible structures are widely used in industrial applications and aerospace structures. ${ }^{1,2}$ Flexible structures have the advantages of light weight and low energy consumption. Nowadays flexible space structures are developing towards the direction of large scale, flexibility, and low stiffness. However, vibration is easily caused by external disturbance due to the low stiffness and small damping of the material. ${ }^{3,4}$ Moreover, a dynamic aeroelastic instability phenomenon results from the interactions between motions of an aircraft panel and aerodynamic loads exerted on that panel by air flowing past one of the faces, which is called panel flutter. ${ }^{5,6}$ Vibration and flutter will lead to unwanted displacements, positioning errors, and even worse, lead to the destructive damage of the structure. ${ }^{2}$ Especially, when the structure vibrates at its resonance frequencies, the structural working life will be greatly shortened. Therefore, active vibration control must be applied to guarantee the normal working of flexible structures.

The vibration problem has motivated a huge amount of research in the vibration control of flexible structures, and a great amount of work has been conducted in the field of smart structures by many researchers. Forward first used the piezoelectric ceramics in the vibration control of an end-supported mast. ${ }^{7}$ Bailey and Hubbard designed an active vibration damper for a cantilever beam using polyvinylidene fluoride (PVDF) film as a distributed-parameter actuator. ${ }^{8}$ An optimal control design is presented by Zhou et al. to actively suppress large-amplitude, limited-cycle flutter motions of rectangular isotropic plates at supersonic speeds using piezoelectric actuators. ${ }^{6}$ The results demonstrate that the piezoelectric materials show good performance in panel flutter suppression. Li used the piezoelectric material to improve the flutter characteristics of the supersonic plates. ${ }^{9}$ The numerical results showed that the aeroelastic flutter properties can be greatly improved by introducing active stiffness and active mass into the supersonic plate with piezoelectric patches.

At present, researches on vibration control mainly lie in modelling methods of the mathematical model, optimal placement of actuators/sensors, and control algorithms. ${ }^{10-12}$ The locations of the actuators/sensors have a significant influence on the performance of the control system. Misplaced actuators/sensors may lead to problems such as lack of observability and controllability. Researchers have used many optimization criteria and techniques to find the optimal location of actuators/sensors. Arbel first proposed the concept of controllability/observability in the optimal placement of actuators/sensors. ${ }^{13}$ Bruant and Proslier proposed a modified optimization criterion in consideration of the spillover effects. ${ }^{14}$ Some researchers have also suggested other optimization criterion based on $H_{2}$ or $H_{\infty}$ norms. ${ }^{15-17}$ There are also many other optimization criterion such as the minimization of the linear quadratic regulator $\operatorname{cost}^{18}$ and the maximization of the harvested strain energy in piezoceramic materials. ${ }^{19}$ In addition, the genetic algorithm (GA) has been extensively used in finding the optimal places of the actuators/sensors. ${ }^{20,21}$

There are many control algorithms utilized to suppress the vibration of flexible structures. Warminski et al. studied four types of control algorithms for the vibration suppression of a large, flexible composite beam structure. ${ }^{22}$ Shin et al. designed an acceleration feedback (AF) controller for a clampedclamped beam. ${ }^{23}$ Lin and Liu presented a novel resonant fuzzy logic controller (FLC) to minimize structural vibration using collocated piezoelectric actuator/sensor pairs. ${ }^{24}$ The experimental results demonstrated the effectiveness of the FLC in active vibration control. Qiu et al. studied a kind of discretetime sliding mode variable structure control (VSC) algorithm to suppress vibration of a flexible plate. ${ }^{25}$ The experiments demonstrated that the proposed control algorithm is feasible 